\title{
Optical Sensor based Efficient Internal Body Organ Monitoring
}

\author{
Muhammad Younus \\ Daffodil International University \\ 102, Sukrabad, Mirpur Road \\ Dhanmondi, Dhaka \\ Bangladesh
}

\author{
Pankaj Chakrabarty \\ Daffodil International University \\ 102, Sukrabad, Mirpur Road \\ Dhanmondi, Dhaka \\ Bangladesh
}

\author{
Mosharrafa Ahmad \\ Daffodil International University \\ 102, Sukrabad, Mirpur Road \\ Dhanmondi, Dhaka \\ Bangladesh
}

\begin{abstract}
The work has been inspired by the need to find an efficient method for detecting internal disorders of body organs (heart) using optical sensor which is simple and the comparatively newer. The initial task is to gather the ideas and modify them. In this work an idea has been given to detect the internal disorder of heart by using the reflection and refraction of optical sensor and ECG. An optical sensor has been used as a detector which collects the initial signals and two parallel glasses to show the refraction of light and ECG to see output. In this process the data of internal part of body can be easily found. Using some equations and data the disorders and disabilities can be observed. Some data has been generated for healthy parts of body and disordered parts. The differences of obtained data show the effective result of this work. Data obtained from electrocardiogram (ECG) signals provides invaluable tools for diagnosing cardiac disorders.
\end{abstract}

\section{General Terms}

Heart monitoring, ECG

\section{Keywords}

Optical Sensor, Detecting ECG

\section{INTRODUCTION}

In previous days it has seen that different types of disorder of body organs detected by the different types of methods. In this thesis it has been shown that how to detect disorder by an optical sensor. An optical sensor has used with inferred rays collects data as a source. The experiment has been running on heart. Data has been collected on the base of heart functions. Firstly a flow chart has shown which has given the working idea instantly. Most information has been given about optical fiber sensor, it's advantages and uses in biomedical sector [1]. Beside them the concept of mirror and concept of heart and ECG elaborately has discussed. By combining the whole of these concepts step by step a structure has made for this thesis. Electrocardiogram (ECG) is a nearly periodic signal that reflected the activity of the heart. A lots of information on the normal and pathological physiology oh heart can be obtained from ECG. However the ECG signals being non stationary in nature, it is very difficult to visually analyze them. Thus there need a computer based method of ECG signal analysis. A lot of works has been done in the field of ECG signals analysis using various approaches and methods. The basic principal of all the methods however involves transformation of ECG signal different transformation techniques including Fourier transform, Hilbert Transform, Wavelet transform etc. Physiological signals like ECG are considered to be a quasi-periodic nature. They are of the finite duration and non stationary. Hence a technique like Fourier series (based on sinusoids of infinite duration) is inefficient for ECG. Here the Fourier transform has used as the simulated process [2].

\section{OPTICAL FIBER SENSOR}

A fiber optic sensor is a sensor that uses optical fiber either as the sensing element ("intrinsic sensors"), or as a means of relaying signals from a remote sensor to the electronics that process the signals ("extrinsic sensors")[ 3].

\subsection{Fiber Optic Sensor Principles}

The general structure of an optical fiber sensor system is shown in bellow figure. It consists of an optical source (Laser, LED, Laser diode etc), optical fiber, sensing or modulator element (which transduces the measurand to an optical signal), an optical detector and processing electronics (oscilloscope, optical spectrum analyzer etc) [3].

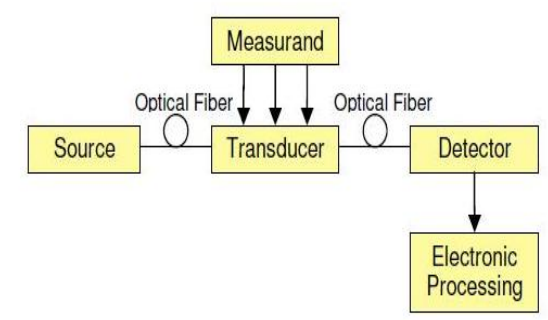

Fig 1: Basic components of an optical fiber sensor system.

\section{HEART}

The heart, located in the mediastinum, is the central structure of cardiovascular system. It is protected by the bony structure of the sternum anteriorly, the spinal column opsteriorly, and the rib cage.

Sinoatrial (SA) node is the dominant pacemaker of heart, located in the upper portion of right atrium. It has intrinsic rate of $60-100 \mathrm{bpm}$.

Artioventicular (AV) node is a part of AV junction tissue. It shows conduction, creating a slight delay before impulse reach ventricles. It has an intrinsic rate of $40-60 \mathrm{bpm}$. [2]. 
Table 1: Depolarization and Repolarization

\begin{tabular}{|l|l|}
\hline Action & Effect \\
\hline Depolarization & $\begin{array}{l}\text { Shifting of electrolytes across the } \\
\text { cell membrane causes change in } \\
\text { the electric charge of the cell. It } \\
\text { results in contraction. }\end{array}$ \\
\hline Repolarization & $\begin{array}{l}\text { Internal negative charge is } \\
\text { restored and the cell returns to } \\
\text { their resting state. }\end{array}$ \\
\hline
\end{tabular}

\section{The 3 stages of a single heart bit are}

1. Atrial depolarisation

2. Ventricular depolarisation

3. Atrial and ventricular re-polarisation.

Normal function of heart simplest way to describe the heart is as a "pump". Quite often doctors and nurses take the analogy further and talk to patients about their "plumbing". This analogy is reasonably accurate.

The role the heart plays in the cardiovascular system is similar to the role played by the pump in your central heating system. The heart pumps blood through the arteries and veins to organs, muscles and tissues, just as the central heating pump forces hot water through the pipes to the radiators. [2].

\subsection{Electrocardiogram (ECG)}

An ECG is a series of waves and deflections recording the heart's electrical activity from a certain view. Many views, each called a lead, monitor voltage changes between electrodes placed in different position on the body. Each cardiac cell each surrounded by and filled with solutions of sodium $(\mathrm{Na}+)$, Potassium $(\mathrm{K}+)$, and calcium $(\mathrm{Ca}+)$. The interior of the cell membrane is considered to be negative with the respect to outside during resting conditions. When an electric impulse is generated in the heart, the interior part becomes positive with respect to exterior. The change of this polarity is called

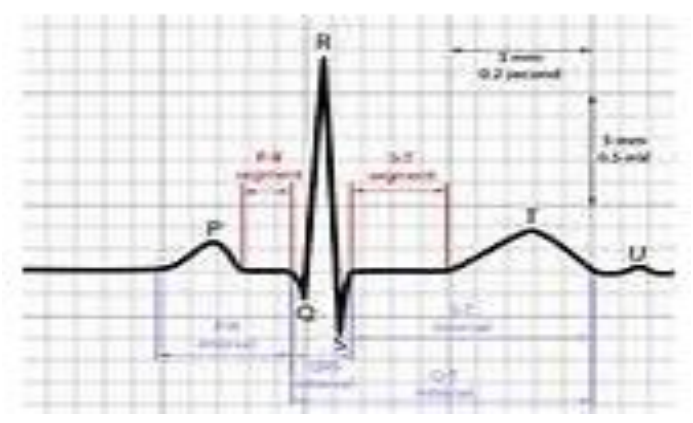

Fig 2: normal heart bit rate depolarization. After depolarization the cell comes back to its original signal of the heart[12].

ECG signals play a vital role in advanced diagnostic methods of various cardiovascular diseases. They provide crucial medical information on the overall health status of a patient. In addition to providing a reliable method for monitoring the electrical cardiac activity, basic aspects of a human physiology such as the Heart Rate Variability (HRV) can be determined from ECG signals .A typical ECG signal shows the oscillations between cardiac contractions (systole) and relaxations (diastole) states as reflected in a heart rate (HR). Thus the ECG signal determines the number of heart beats per minute. A number of important events characterize cardiac functions. Atrial and ventricular depolarization/re-polarization takes place for each heart beat. The cardiac cycle is associated with portions of the heart becoming positively charged, while the remaining parts become negatively ventricular depolarization/re-polarization takes place for each heart beat. The cardiac cycle is associated with portions of the heart becoming positively charged, while the remaining parts become negatively charged interchangeably. This potential difference generated initiates the flow of current. A typical ECG signal depicts a series of waveforms which occur in a repetitive order. The waveforms are initiated from the isometric line, from which a deflection indicates electrical activity. The principal features of an ECG signal, depicted by troughs and peaks, usually denoted by letters $\mathrm{P}, \mathrm{Q}, \mathrm{R}, \mathrm{S}$ and $\mathrm{T}$, are illustrated in Figure 1. One normal heart beat is represented by a set of three recognizable waveforms that start with the P-wave, followed by the QRS complex and ends with the T-wave. The relatively small P-wave is initiated by the depolarization of the atrial muscles and is related to their contraction. The large QRS-wave complex, made up of three waves, is caused by the depolarization of the ventricles and is connected to their contraction.

Atrial re-polarization happens during the depolarization of the ventricles but its weak signal is undetected on an ECG. The Twave is caused by currents flowing during the repolarization of the ventricles. A normal cardiac cycle of an individual at rest consisting of all wavefECG signals are generated using electrocardiographs. Such signals are usually vitiated by several sources of noise which include (i) electrical interference from surrounding equipment (e.g. effect of the electrical mains supply), (ii) measurement (or electrode contact) noise, (iii) electromyographic (muscle contraction), (iv) movement artifacts, (v) baseline drift and respiratory artifacts and (vi) instrumentation noise (such as artifacts from the analogue to digital conversion process). One method of dealing with corrupt ECG signals is through the use of signal filtration systems .ohms (form $\mathrm{P}-\mathrm{T}$ waves) sp charged interchange $8 \mathrm{sec}$. [16].

\section{WORKING PROCEDURE}

The working flow chart of this work has given bellow:

In body part an optical sensor has been connected. Then from heart a bioelectric signal has been generated. The signal passes through the optical fiber where the signal converted into light signal. The light signal reflected by two parallel mirrors those are put one is in 90 degree and 


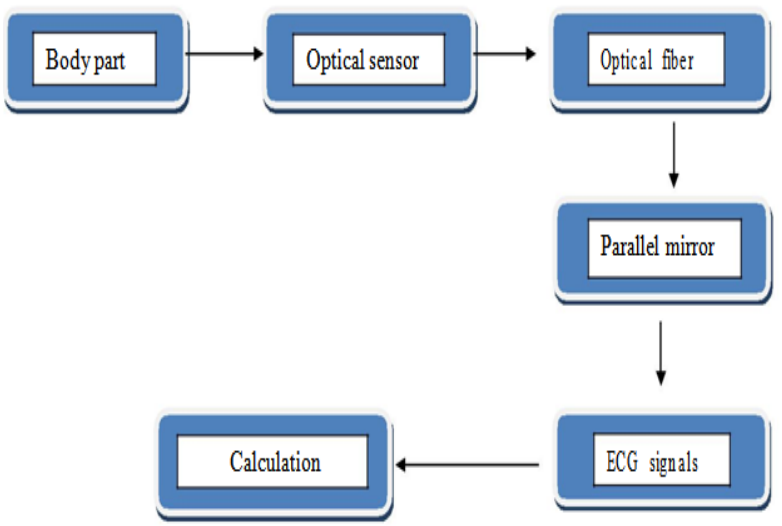

Fig 3: Block diagram of propose method.

another one is in 45 degree angle. The reflected signal has been converted again into electric signal. This electric signal has been worked as the ECG input. Then using this electric signal the simulation part has been completed. In the simulation part by using an artificial ECG signal the heart beat rate has calculated. After changing the heart bit rate and peak voltage some graphs and data has been generated which shows the differences of healthy heart and disordered heat.

\section{SIMULATION AND RESULS}

We use the synecg to generate 10-seconds synthetic ECGs for a number of heart rates of a resting individual with a peak voltage of $1.2 \mathrm{mV}$. illustrates how the function is summoned in the MATLAB command window; the figures show the artificial ECGs generated for $60 \mathrm{bpm}, 70 \mathrm{bpm}, 80 \mathrm{bpm} 90 \mathrm{bpm}$, $50 \mathrm{bpm}$ and $95 \mathrm{bpm}$ heart rates at a desired peak voltage of $1.2 \mathrm{mV}$. We note that heart beats in a regular rhythm is usually between 60 and 100, that is when the signal to noise ratio is usually quite good in a person at rest. Basically, the ECG is a piecewise continuous graph of potential difference (in $\mathrm{mV}$ ) against time (in seconds). The Synthetic ECG generated for 95bpm displays approximately 17 cardiac cycles in 10 seconds. The results are shown below

Table 2: Number of heart rate and Peak voltage

\begin{tabular}{|l|l|l|l|}
\hline Serial no & $\begin{array}{l}\text { Heart bit } \\
\text { rate (bpm) }\end{array}$ & $\begin{array}{l}\text { Peak } \\
\text { voltage } \\
(\mathrm{mV})\end{array}$ & $\begin{array}{l}\text { Bitrate per } \\
10 \mathrm{sec} .\end{array}$ \\
\hline 1 & 60 & 3 & 10 \\
\hline 2 & 70 & 5 & 13 \\
\hline 3 & 80 & 6 & 13 \\
\hline 4 & 90 & 6 & 14 \\
\hline 5 & 50 & 2 & 9 \\
\hline 6 & 95 & 6 & 17 \\
\hline
\end{tabular}

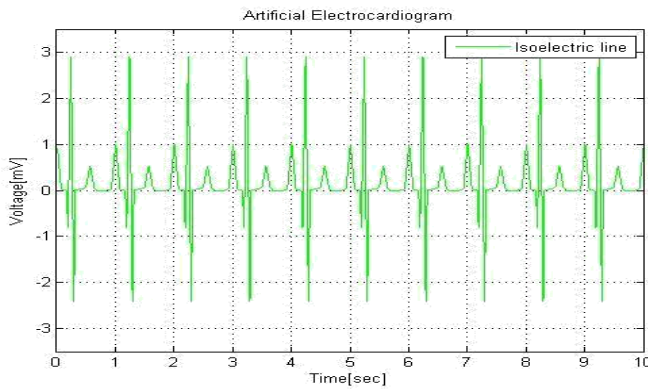

Fig 4: for heart rate 60bpm and Peak voltage $3(\mathrm{mv})$.

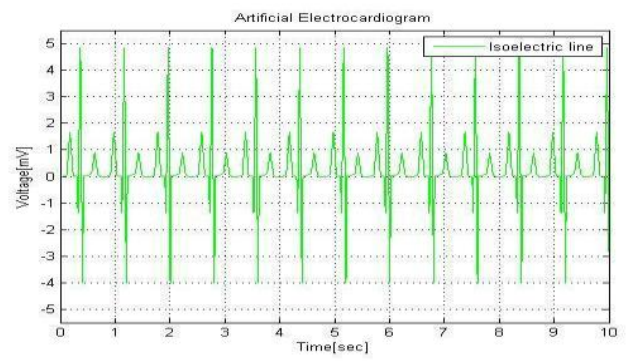

Fig 5: for heart rate 70bpm and Peak voltage $5(\mathrm{mv})$.

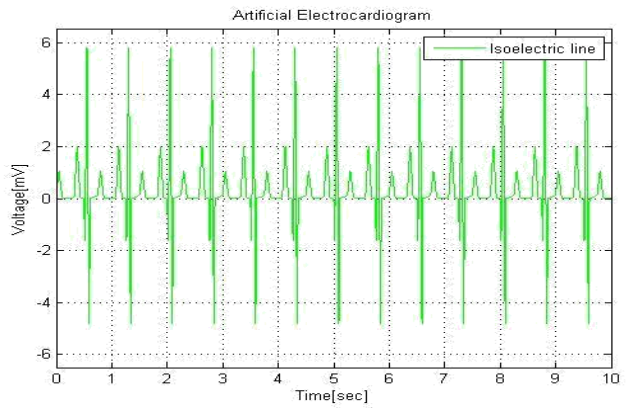

Fig 6: for heart rate 80bpm and Peak voltage $6(\mathrm{mv})$.

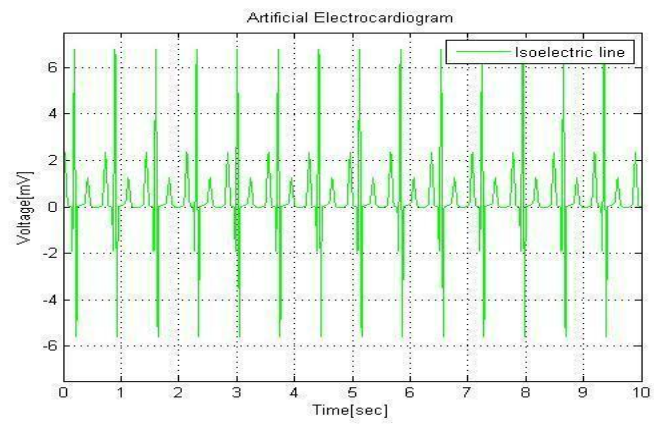

Fig 7: for heart rate $90 \mathrm{bpm}$ and Peak voltage 6 (mv).

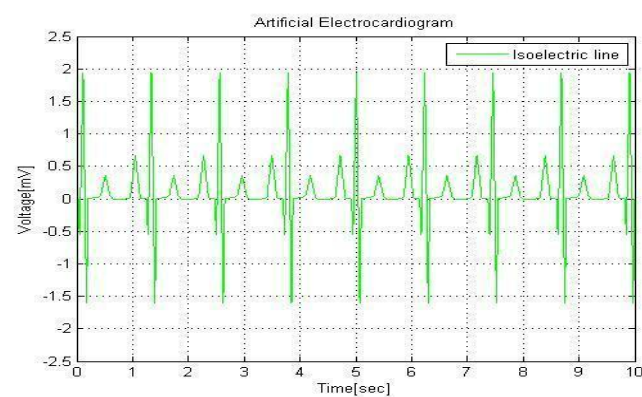

Fig 8: for heart rate 50bpm and Peak voltage $2.5(\mathrm{mv})$. 


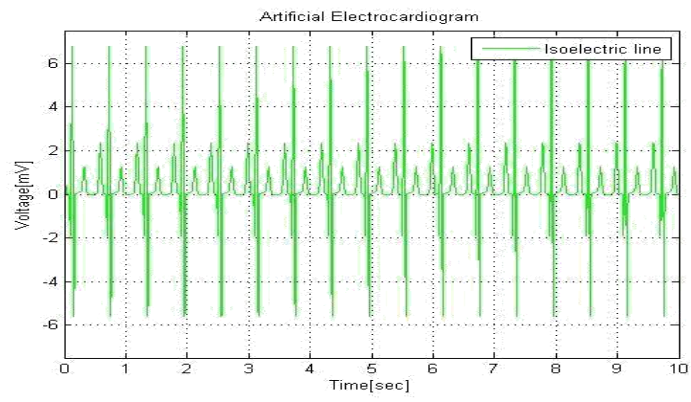

Fig 9: for heart rate 90bpm and Peak voltage $6(\mathrm{mv})$.

With the synecg, we have been able to generate a 10 -seconds artificial ECG for some selected heart rates. The $60 \mathrm{pbm}$ generated 10-seconds ECG displays an approximate 10 cardiac cycles. As heart rate increases, the number of approximate cardiac cycles in the 10-seconds ECG also increases. Variations the heart rates time intervals can be monitored with this increase in heart rate. A careful observation of the $60 \mathrm{bpm}$ ECG generated by synecg reveals that one cardiac cycle takes 0.8 seconds as human physiology suggests. We observe from the generated ECGs that as heart rate increases; the time taken for a cardiac cycle reduces. Similar observations were made for $70 \mathrm{bpm}, 80 \mathrm{bpm}, 90 \mathrm{bpm}$, $50 \mathrm{bpm}$ and $95 \mathrm{bpm}$. The various waveforms which constitute a cardiac cycle vary in length as heart rates change from time to time for the resting individual.

\section{CONCLUTION}

With the synecg function, synthetic ECG signals of various heart rates can be generated of the heart. The function has made use of the function "sgolay" which is specifically geared towards ECG noise reduction and uses Savitzy-Golay polynomial filtering which is well-suited to smoothing of ECG data and preserve the peaks and valleys of the ECG signals better than a standard FIR filter. The thesis have been able to show that the function generated synthetic ECG for 10 seconds by inputting heart rate (per minute) and the desired peak in millivolts. Here the propose has been shown that the method has been applied is more economic advantage than other methods. The heart rate input are as follows; 50bpm, $60 \mathrm{bpm}, 65 \mathrm{bpm}, 70 \mathrm{bpm}, 80 \mathrm{bpm}$ and $95 \mathrm{bpm}$. In all cases, we observe the number of cardiac cycles in the time duration. Here several factors have attributed. To help solve these problems, a simple but inexpensive and easy-to-implement has developed in MATLABTM model that generates ECG signals and gives us mathematical control over the ECG signal. Our model fuses Mathematical functions in MATLABTM with physiological data.

\section{FUTURE WORK}

In the thesis an idea has been given that how to calculate and monitor the disorder of heart by using the optical sensors, parallel mirror and ECG signal using by MATLAB. But it is only an initial part where the idea has been given up. Since inputs to obtain an ECG signal is just the heart beat rate. The future prospects of this function are many. Synecg can be used to further develop a complete dynamic signal monitoring software for the electrical activity of the heart. This is an example of how Mathematical principles can be used in the dynamic modeling of biological rhythms of the human body as in the field of Biomathematics and biomedical sector. So in future the whole idea will be good and interesting area for research and implement for detecting the disorders of body organs.

\section{REFERENCE}

[1] R S Khandpur, "Handbook of Biomedical Instrument", $2^{\text {nd }}$ edition

[2] Md Shoib Farhan, Md kamrul hossain, Asker Ibnea Shaikh, "Determination of r-peak of ECG signal and heart rate using electrical mode decomposition". December, 2010.

[3] Wikipedia, Free Encyclopedia.

[4] Roland, U.; et al. (2003). "A New Fiber Optical Thermometer and Its Application for Process Control in Strong Electric, Magnetic, and Electromagnetic Fields". Sensor Letters 1: 93-8.

[5] Roland, U.; et al. (2003). "A New Fiber Optical Thermometer and Its Application for Process Control in Strong Electric, Magnetic, and Electromagnetic Fields". Sensor Letters 1: 93-8.

[6] "An Integrated System for Pipeline Condition Monitoring" Retrieved 2010-09-22.

[7] Kouznetsov, D.; Moloney, J.V. (2003). "Highly efficient, high-gain, short-length, and power-scalable incoherent diode slab-pumped fiber amplifier/laser". IEEE Journal of Quantum Electronics 39 (11): 1452-1461

[8] Jay M. Enoch, School of Optometry, "History of Mirrors Dating Back 8000 Years" University of California at BerkeleyPer Hadsund, "The Tin-Mercury Mirror: Its Manufacturing Technique and Deterioration Processes", Studies in Conservation, Vol. 38, No. 1 (Feb., 1993)

[9] Lekner, John (1987). Theory of Reflection, of Electromagnetic and Particle Waves. Springer. ISBN 9789024734184 .

[10] Mandelstam, L.I. (1926). "Light Scattering by Inhomogeneous Media". Zh. Russ. Fiz-Khim. Ova. 58: 381

[11] "Shoaling, Refraction, and Diffraction of Waves". University of Delaware Center for Applied Coastal Research. Retrieved 2009-07-23.

[12] Yun Sun ,Cap luck Chan, Sankar Muthu Krisnan, "ECG signal conditioning by morphological filtering", Computers in biology and medicine 32 (2002)

[13] Fokko Jan Dijksterhuis (2004). Lenses and Waves: Christiaan Huygens and the Mathematical Science of Optics in the Seventeenth Century. Springer. ISBN 14020-2697-8.

[14] John D Joannopoulos, Johnson SG, Winn JN \& Meade RD (2008). Photonic Crystals: Molding the Flow of Light (2nd ed.). Princeton NJ: Princeton University Press. ISBN 978-0-691-12456-8

[15] Andrew S. Glassner (1989). An Introduction to Ray Tracing. Morgan Kaufmann.ISBN 0-12-286160-4.

[16] Joseph Ackora-Prah, Anthony Y. Aidoo, Kwasi Baah Gyamf, "An Artificial ECG Signal Generating Function in MATLABTM", Applied Mathematical Sciences, Vol. 7, 2013, no. 54, 2675 - 2686 